Physics A, 27, (1994), 6157-6166

\title{
STABILITY ESTIMATE IN SCATTERING THEORY AND ITS APPLICATION TO MESOSCOPIC SYSTEMS AND QUANTUM CHAOS
}

\author{
Alexander G. Ramm ${ }^{1}$ and Gennady P. Berman ${ }^{2,3}$ \\ ${ }^{1}$ Department of Mathematics, \\ Kansas State University, Manhattan, KS 66505-2602, U.S.A. \\ E-MAIL: ramm@math.ksu.edu \\ ${ }^{2}$ Center for Nonlinear Studies, MS-B258 \\ Los Alamos National Laboratory \\ Los Alamos, New Mexico 87545, U.S.A. \\ E-MAIL: GPB@GOSHAWK.LANL.GOV \\ and \\ ${ }^{3}$ Kirensky Institute of Physics; \\ Research and Educational Center for Nonlinear Processes \\ at The Krasnoyarsk Technical University; \\ Theoretical Department \\ at The Krasnoyarsk State University; \\ 660036, Krasnoyarsk, Russia
}




\begin{abstract}
We consider scattering of a free quantum particle on a singular potential with rather arbitrary geometry of the boundaries of its support. In the classical limit $\hbar=0$ this problem reduces to the well known problem of chaotic scattering. The universal estimates for the stability of the scattering amplitudes are derived. The application of the obtained results to the mesoscopic systems and quantum chaos are discussed. We also discuss a possibility of experimental verification of the obtained results.
\end{abstract}




\section{Introduction}

Recently much attention has been paid to the theoretical and experimental investigations of the scattering of a free quantum particle on the obstacles with rather complicated form of boundaries. Of special interest are the studies of the scattering processes of an electron with the mesoscopic resonant tunneling structures, when quantum effects and the geometry of the scattering potential are important [1-13]. Usually, these quantum systems are nonintegrable, which means that the number of degrees of freedom exceeds the number of global independent integrals of motion. In this case one can not separate variables in the Schrödinger equation, and in the corresponding Newtonian equations. Treated classically these systems exhibit dynamical chaos, that is, strong (exponential) instability of motion under small variation of parameters (such as energy of an incident wave, form of the potential, etc). That is why one of the main problems in studying such systems is to determine the role and contribution of fluctuations and correlations in the scattering amplitudes and cross sections [14-22].

The problem of fluctuations of the scattering amplitudes and cross sections in the processes of elastic (and inelastic) collisions is well known, and has a long history (see [23-28] and references therein). In the elastic scattering, the fluctuations of the scattering amplitudes can appear because of a high sensitivity to the details of the scattering: the parameters of the incident wave and the geometry of the scatter potential. At the same time, the coherent effects (correlations) are also present in the scattering processes in some region of parameters $[21,22,23,24,29]$. Thus, the problem arises: how does one separate and describe the random and the coherent effects in the scatttering processes, and how does one measure their contribution in exper- 
iments?

The first theoretical investigations of the statistical properties (fluctuations) of the scattering amplitudes and cross sections were presented in [23-28] (Ericson fluctuations). According to [23,24], the main reasons why the scattering amplitudes become random are the following. Let an incident wave have a wave-length $\lambda=2 \pi / k$ much smaller than the characteristic dimension $L$ of the region $D$ where the scattering potential $q(x)$ (see below) is located: $k L \gg 1$, and $x=\left(x_{1}, \ldots, x_{n}\right)$. Before escaping the region $D$, the incident wave can be reflected a large number of times from the boundaries $\Gamma_{j}$ of the support of the potential $q(x)$. In this case, a wave close to a standing wave appears in the system. These "quasi-standing" (or quasy-stationary) waves can be associated with the resonances in the scattering amplitude. These resonances are observed in various experiments, and recently in very promising for the future applications mesoscopic systems (see, for example, [30-32]). Each $n$-th resonance is characterized by two main parameters: the energy $E_{n}$, and the width $\Gamma_{n}$ [33]. There is usually one more important parameter which characterizes the spacing between the neighboring resonances: $\Delta E_{n}$. Because the process of scattering is completely defined, the scattering amplitudes should be reproducible in different experiments, provided that all conditions remain identical. However, as was mentioned above, under the condition $k L \gg 1$ the number of reflections of the incident wave in the region $D$ can be very large (in $[23,24]$ also the following inequality is assumed to be satisfied: $\Gamma_{n} / \Delta E_{n} \gg 1$, which is called the regime of overlapping levels). Then, a small variation of parameters can completely change the "trajectory" of the wave, and consequently, the phase of the scattering amplitude. These ideas were developed in $[23,24,26]$ on the basis of the statistical approach [34]. 
Recently, the problem of fluctuations of the scattering amplitudes has attracted additional interest in connection with the so-called "chaotic (irregular) scattering" (CS) in chemical reactions, particle physics, mesoscopic systems and other areas of physics [14-22,35-37]. The investigations of the CS can be conventionally divided into three groups: (1) classical CS (CCS), (2) semiclassical CS (SCS), (3) quantum CS (QCS). The basic ideas are associated with the CCS, since only in this case the "real dynamical chaos" occurs. The investigations of the CCS were stimulated by the significant progress achieved recently in studying of the dynamical chaos in the classical bounded Hamiltonian systems [38-41]. The classical phase space in this case can be very complicated, and each of the trajectories belongs to one of the following three classes: (a) stable periodical trajectories, (b) unstable periodical trajectories, (c) chaotic (unperiodical) trajectories. Dynamical chaos in bounded systems is stationary in the sense that it does not disappear at large times $(T \rightarrow \infty)$. The systems where the CCS takes place are unbounded, and the additional trajectories appear: (d) unbounded trajectories. For this trajectories chaos can be only transient.

A special interest in the scattering problem represent singular potentials $q(x)$ which satisfy the following property: $q(x)=\infty$ when $x \in D$, and $q(x)=0$ when $x \notin D$, where $D$ is some compact (located region) in $R^{n}$. In the case of a singular potential $q(x)$ considered below, the trajectories (a) can be absent (see, for example, [15]), and the trajectories (b) and (c) represent a so-called "repeller" $\Omega_{R}[15]$. For the trajectories $(\mathrm{d})$ this repeller leads to the "transient chaos" which was previously investigated in various bounded conservative and dissipative systems (see, for example, [42-44]). In this sense a singular scattering potential leads to the mostly chaotic classical "repeller", and to the biggest level of fluctuations in the scattering amplitude. 
The main achievements in the CCS are associated with the understanding of the following facts: (1) although, in the CCS a direct contribution in the cross section is connected with the trajectories $(d)$, the influence of the repeller $\Omega_{R}$ - bounded (trapped) trajectories on the process of scattering and fluctuations plays a very important role; (2) the CCS is a general phenomenon rather then an exception. (In some special cases of a singular potential [45], the set $\Omega_{R}$ can consist of only one unstable periodic trajectory). Usually, for singular potentials, a repeller $\Omega_{R}$ is a Cantor set with a fractal structure (see, for example, paper [15] where an elastic scattering on three hard discs (3HD) was considered), and is characterized by several quantities, such as the Hausdorff dimension $D_{H}$, Lyapunov exponents $\lambda_{i}$, the Kolmogorov-Sinai entropy per unit time $h_{K S}$, the escape rate $\gamma$, and other quantities (see [15] and references therein). There are some relations between these parameters, for example, (see [15]):

$$
\gamma=\sum_{\lambda_{i}>0} \lambda_{i}-h_{K S}
$$

The escape rate $\gamma$ is a classical equivalent of the resonance width $\Gamma: \gamma \sim \Gamma / \hbar$ [15]. So, the relation (1.1) shows a fundamental property of the CCS: when a repeller $\Omega_{R}$ is chaotic $\left(h_{K S}>0\right)$, the escape rate (and the resonance width $\Gamma)$ is decreasing. Also, in this case large fluctuations appear in the quantities which characterize the process of CCS, for example, in the time delay function $[15,20,22]$.

When one investigates the SCS and the QCS, the main problem is: what are the "fingerprints" of the classical chaos on the quantum scattering ? For the first time, the problem of QCS was considered in [14], where the elastic scattering was studied on a two-dimensional surface of a constant negative curvature. According to [14], the scattering phase shift as a function of the 
momentum is given by the phase angle of the Riemann's zeta function, and displays a very complicated (chaotic) behavior (see for details $[14,21,22]$ ). In [16] the SCS was studied in the system of 3HD using the analysis based on the Gutzwiller trace formula [46]. This trace formula is valid when all periodic orbits of the repeller $\Omega_{R}$ are unstable and isolated. Both these conditions can be satisfied for the singular potential $q(x, t \rightarrow \infty)$ considered in sections 2 and 3 , including a particular case of a singular potential of the 3HD system considered in [15-18].

The quantum analysis presented in $[21,22]$ shows that in the QCS the statistical properties of the fluctuations in the cross section can be described by the theory of random matrix ensembles [41]. Different aspects on the problem of fluctuations in the SCS and QCS are discussed in [14,16-28,35$37]$.

At the same time, much less is known about the contribution and characterisic properties of the correlations (coherent component) in the chaotic scattering. As was pointed out in $[23,24]$, a significant level of correlations in the cross section should be expected when, for example, the energy change $\delta E$ of the incident wave in (2) is small compared with the resonance width $\Gamma(\Gamma / \delta E>1)$. According to $[23,24]$, in this case essentially the same states are exited, and the scattering amplitudes are changed insignificantly. The existence of correlations in the QCS was discussed also in [21,22] for some quasi-1D periodical potential (in [22] also an experiment is discussed in connection with the correlations in the chaotic scattering). It was shown in $[21,22]$ that the correlations in energy for the matrix elements of the $S$ matrix exist, and exhibit themselves when $\Gamma / \delta E>1$, in agreement with the Ericson hypothesis $[33,34]$.

The same problem on the contribution of correlation and fluctuation ef- 
fects arises when calculating a transition probability of an injected electron transmitted through mesoscopic devices such as double-barrier resonance tunneling structure (DBRTS), quantum dots and others [8-13, 30-32]. In this case a transmitted electron "feels" the boundary of the scattering potential $q(x)$, and the transmission amplitude can vary significantly depending on the small variation of the sample's form. As it was discussed above, we again come in this case to the problem of chaotic scattering. So, both these problems, quantum chaotic scattering and the scattering problem in mesoscopic systems are srtrongly connected. The correlation properties of the transmission amplitude in mesoscopic system, at rather big values of $\Gamma$, were observed in the experiment [32].

In this situation it is important to consider the problem of contribution of correlations and fluctuations to the quantum chaotic scattering for a rather general class of potentials $q(x)$. Although, in this case only general statements about the scattering amplitude can be made, such an approach has an obvious advantage: these general statements can be applied to a wide variety of systems. From this poin of view singular potentials are of particular interest, because they may produce the "repeller" $\Omega_{R}$ which can be "extremely chaotic", and the level of fluctuations can significantly increase. There are no results allowing to estimate the contribution of fluctuations and correlations in the scattering amplitude in this case. Our main result discussed below, says is that even in the case of strongly singular potentials some universal correlations exist in the scattering amplitude.

In this paper we consider a scattering problem for a free quantum particle scattered by a bounded obstacle with rather arbitrary shapes of the boundary. The boundary may consist of several connected components. As was already mentioned, similar situation occurs in the processes of ballistic 
scattering in the mesoscopic systems widely considered nowadays. The results obtained in this paper can be formulated as follows. It is shown that there exists a region of parameters where small variation of rather arbitrary strongly singular potential (Note, that the variation of the total energy is infinite for the potentials we consider) leads only to small variations of the scattering amplitudes. This region of parameters can be defined as a region of strong correlations. These correlations are universal in the sense that they do not depend on the concrete structure of the resonances in the scattering amplitude in a particular system under consideration. Because the results we discuss in this paper are given in the form of the exact statements, we outline their proofs.

The paper is organized as follows. In section 2 we present a stability estimate for the scattering amplitudes for a rather wide class of potentials. In section 3 a proof of the stability of the scattering amplitudes is given for a singular potential. Discussion of the results is given in section 4 .

\section{Stability Estimate for the Scattering Amplitude}

In this section we prove that small variations of the potential lead to small perturbations of the scattering amplitude for a class of strongly singular potentials which can take infinite values on sets of positive measure. The notion of small variations will be specified.

1. Let $D=\bigcup_{j=1}^{J} D_{j}, \Gamma:=\partial D=\bigcup_{j=1}^{J} \Gamma_{j}$, where $D_{j} \subset R^{n}$ is a bounded domain with a $C^{2, \nu}, 0<\nu \leq 1$, boundary $\Gamma_{j}$. This means that in the local coordinates the equation of $\Gamma_{j}:=\partial D_{j}$ is $x_{n}=\phi\left(x^{\prime}\right), x^{\prime}:=\left(x_{1}, x_{2}, \ldots, x_{n-1}\right)$, $\phi \in C^{2, \nu},\|\phi\|_{C^{2, \nu}} \leq \Phi_{\nu}$.

Assume $D \subset B_{a}:=\{x:|x| \leq a\}$, and $D_{j} \cap D_{i}=\emptyset$ if $i \neq j, J<\infty$. 
Define $u_{0}:=\exp (i k \alpha \cdot x)$ and

$$
q(x ; t):=t \chi_{D}(x), \quad \chi_{D}(x):= \begin{cases}1, & \text { in } D \\ 0, & \text { in } D^{\prime}:=R^{n} \backslash D\end{cases}
$$

where parameter $t \in[1, \infty]$. For definiteness take only $n=3$ in what follows. Consider the scattering problem

$$
\begin{gathered}
{\left[\nabla^{2}+k^{2}-q(x ; t)\right] u=0 \quad \text { in } R^{3} .} \\
u=\exp (i k \alpha \cdot x)+A^{(t)}\left(\alpha^{\prime}, \alpha, k\right) \frac{\exp (i k r)}{r}+o\left(\frac{1}{r}\right), \\
r:=|x| \rightarrow \infty, \quad \frac{x}{|x|}:=\alpha^{\prime} .
\end{gathered}
$$

The scattering solution $u(x, \alpha, k ; t):=u(t)$ is uniquely defined as the solution of $(1),(2)$. It was proved in [47-49], that

$$
\left|u(t)-u_{\Gamma}\right| \rightarrow 0, \quad \text { as } \quad t \rightarrow+\infty
$$

where $u_{\Gamma}$ is the scattering solution to the obstacle scattering problem

$$
\begin{gathered}
\left(\nabla^{2}+k^{2}\right) u_{\Gamma}=0 \quad \text { in } D^{\prime}, \quad u_{\Gamma}=0 \quad \text { on } \Gamma, \\
u_{\Gamma}=u_{0}+A_{\Gamma}\left(\alpha^{\prime}, \alpha, k\right) \frac{\exp (i k r)}{r}+o\left(\frac{1}{r}\right), \quad r=|x| \rightarrow \infty, \quad \alpha^{\prime}:=\frac{x}{r} .
\end{gathered}
$$

The relation (2.3) has the following meaning

$$
\begin{gathered}
\|u(t)\|_{L^{2}(D)} \leq \frac{c}{\sqrt{t}}, \quad\left\|u(t)-u_{\Gamma}\right\|_{H^{2}\left(\tilde{D}^{\prime}\right)} \leq \frac{c}{t^{1 / 4}}, \\
\|u(t)\|_{L^{2}(\Gamma)} \leq \frac{c}{t^{1 / 4}}
\end{gathered}
$$

where $\tilde{D}^{\prime}$ is any compact strictly inner subdomain of $D^{\prime}$. Here and below $c>0$ denote various positive constants independent of $t$ or other parameters which vary. 
Estimates $(2.6),(2.7)$ are proved in [47-49]. It is proved in [50] that if $q_{j}(x), j=1,2$, generate the scattering amplitudes $A_{j}\left(\alpha^{\prime}, \alpha, k\right)$, then, the following relation holds

$$
-4 \pi A\left(\alpha^{\prime}, \alpha, k\right)=\int_{R^{3}} p(x) u_{1}(x, \alpha, k) u_{2}\left(x,-\alpha^{\prime}, k\right) d x,
$$

where

$$
A:=A_{1}-A_{2}, \quad p:=q_{1}-q_{2},
$$

and $u_{j}$ is the scattering solution corresponding to $q_{j}$. Formula (2.8) is derived in [50] under the assumption that $q_{j}(x) \in L_{l o c}^{p}, p>n / 2$, and $q(x)$ is in $L^{1}\left(B_{R}^{\prime}\right)$, where $B_{R}^{\prime}:=R^{3} \backslash B_{R}, B_{R}:=\{x:|x| \leq R\}, R>0$ is an arbitrary large fixed number.

In [51] an analog of (2.8) is derived for obstacle scattering. Namely, it is proved in [51] that if $\Gamma_{j}, j=1,2$, are bounded sufficiently smooth (say, Lipschitz) surfaces, and $A_{j}$ are the corresponding scattering amplitudes, $A_{j}:=A_{\Gamma_{j}}, A:=A_{1}-A_{2}$, then [51, formula (4)]

$$
\begin{gathered}
-4 \pi A\left(\alpha^{\prime}, \alpha, k\right) \\
=\int_{\Gamma_{12}}\left[\bar{u}_{1 N}(s, \alpha, k) u_{2}\left(s,-\alpha^{\prime}, k\right)-u_{1}(s, \alpha, k) u_{2 N}\left(s,-\alpha^{\prime}, k\right)\right] d s,
\end{gathered}
$$

where $N$ is the exterior unit normal to $\Gamma_{12}=\partial D_{12}$, where $D_{12}:=D_{1} \cup D_{2}$. 2. We claim that, uniformly in $t_{1}, t_{2} \in[1, \infty]$, the following stability estimate holds

$$
\begin{gathered}
\sup _{\alpha^{\prime}, \alpha \in S^{2} ; 0<k_{1} \leq k \leq k_{2}<\infty}\left|A_{D_{1}}^{\left(t_{1}\right)}\left(\alpha^{\prime}, \alpha, k\right)-A_{D_{2}}^{\left(t_{2}\right)}\left(\alpha^{\prime}, \alpha, k\right)\right| \\
\leq c\left\{\left[\min \left(t_{1}, t_{2}\right)\right]^{-1 / 4}+\rho\left(D_{1}, D_{2}\right)\right\},
\end{gathered}
$$

where $c=$ const $>0, c$ is independent on $t_{j} \in[1, \infty]$, and on $D_{j} \subset B_{a}$, $j=1,2$, such that the boundaries of $D_{j}$ satisfy the estimate $\left\|\phi_{j}\right\|_{C^{2, \nu}} \leq \Phi_{\nu}$. 
The distance $\rho\left(D_{1}, D_{2}\right)$ in $(2.11)$ is defined by the formula

$$
\rho\left(D_{1}, D_{2}\right):=\sup _{x \in \partial D_{1}} \inf _{y \in \partial D_{2}}|x-y|
$$

3. Note, that if $t \in\left[1, t_{0}\right]$, where $1<t_{0}<\infty$ is any fixed number, then the following estimate can be derived from (2.8)

$$
\begin{gathered}
\sup _{\alpha^{\prime}, \alpha \in S^{2} ; 0<k_{1} \leq k \leq k_{2}<\infty}\left|A_{D_{1}}^{\left(t_{1}\right)}\left(\alpha^{\prime}, \alpha, k\right)-A_{D_{2}}^{\left(t_{2}\right)}\left(\alpha^{\prime}, \alpha, k\right)\right| \\
\leq \frac{c}{4 \pi}\left|t_{1}-t_{2}\right| \int_{D_{1} \bigcap D_{2}} d x+\frac{c t_{0}}{4 \pi} \int_{D_{12} \backslash\left(D_{1} \bigcap D_{2}\right)} d x \\
\leq \frac{c}{4 \pi}\left|t_{1}-t_{2}\right|\left|D_{1} \bigcap D_{2}\right|+\frac{c t_{0}}{4 \pi}\left\{\left|\partial D_{1}\right|+\left|\partial D_{2}\right|\right\} \rho\left(D_{1}, D_{2}\right) \\
\leq c\left\{\left|t_{1}-t_{2}\right|+\rho\left(D_{1}, D_{2}\right)\right\} .
\end{gathered}
$$

Here we have used the known estimate [50], [52]

$$
\max _{x \in R^{3} ; \alpha \in S^{2} ; 0<k_{1} \leq k \leq k_{2}<\infty}\left|u_{j}\right| \leq c .
$$

In (2.12) $\left|\partial D_{j}\right|$ denotes the area of the surface $\partial D_{j}$, and $\left|D_{1} \cap D_{2}\right|$ denotes the volume of $D_{1} \cap D_{2}$.

4. If $t_{1}=t_{2}=+\infty$, then, the stability estimate

$$
\sup _{\alpha^{\prime}, \alpha \in S^{2} ; 0<k_{1} \leq k \leq k_{2}<\infty}\left|A_{1}\left(\alpha^{\prime}, \alpha, k\right)-A_{2}\left(\alpha^{\prime}, \alpha, k\right)\right| \leq c \rho\left(D_{1}, D_{2}\right)
$$

follows from formula (2.10), since

$$
\begin{gathered}
\sup _{s \in \Gamma_{j} ; \alpha \in S^{2} ; 0<k_{1} \leq k \leq k_{2}<\infty}\left|u_{j N}(s, \alpha, k)\right| \leq c, \\
\sup _{\alpha^{\prime} \in S^{2} ; s \in \Gamma_{j+1} ; 0<k_{1} \leq k \leq k_{2}<\infty}\left|u_{j}\left(s,-\alpha^{\prime}, k\right)\right| \leq c \rho\left(D_{1}, D_{2}\right) .
\end{gathered}
$$

Here $\Gamma_{3}:=\Gamma_{1}, j=1,2$. 
The basic result (2.11), which contains both stability estimates (2.12) and (2.14), is of interest because the inequality (2.11) holds uniformly in $t$, $t \in[1, \infty]$.

5. As an example, we present here the results on the dependence $c(k)$ in (2.14) for the special case of the scattering potential. We claim that the constant $c$ in (2.14) is of the order $O\left(k^{2}\right)$ as $k$ goes to infinity, under the following assumptions: i) $J=1$, ii) $s \cdot N>b>0$ for $s$ in $S_{1}\left(S_{1}:=\Gamma\right)$ and for $s$ in the perturbed surface, say $S_{2}$; here $N$ is the outer normal to $S_{1}$ (or $S_{2}$ ) at the point $s, b>0$ is a constant independent of $s, k$ and other parameters.

Proof of the claim: If ii) holds, then from the estimate (2.6) in [53,p.66] it follows that $\|v\|_{B_{R}}<c, c$ is always assumed to be independent of $k$, $v:=u-u_{0}$, where $u$ is the scattering solution corresponding to $S_{1}$, and $u_{0}$ is the plane wave. From this and the Helmholtz equation one gets $\|v\|_{2}<c k^{2}$, where $\|v\|_{2}$ is the Sobolev space $H^{2}$ norm. Let $\left|v_{N}\right|$ stay for the $L^{2}\left(S_{1}\right)$ norm of $v_{N}$ on $S_{1}$. Then, an interpolation inequality yields the desired estimate: $\left|v_{N}\right|<c k^{3 / 2}$. This estimate implies the claim that the constant $c$ in $(2.14)$ is of the order $O\left(k^{2}\right)$ as $k$ grows to infinity. Indeed, estimating integrals in (2.10) by Cauchy's inequality one gets the sum of the products of the terms of the type $\left|v_{N}\right||v|$ and terms of lower order in $k$ which are easy to estimate by $O\left(k^{3 / 2}\right)$. By an interpolation inequality, the norm $|v|$ is $O\left(k^{1 / 2}\right)$, so the result follows. Let us formulate the known interpolation inequalities used above (see [40])

$$
\left\|D^{r} v\right\|_{L^{2}\left(S_{1}\right)}<c t^{3 / 2-r}\|v\|_{2}+t^{-1 / 2-r}\|v\|
$$

where $\|v\|$ is the $L^{2}$ norm in $B_{a} \backslash D, \partial D=S_{1}, t>0$ in (2.15) is an arbitrary parameter, and $r=0$ or 1 . Take $r=0$ in (2.15) and minimize in $t>0$ the 
right-hand side of (2.15), using the formulas $\|v\|_{2}<c k^{2},\|v\|<c$, to get for the right-hand side the estimate $O\left(k^{1 / 2}\right)$. Similar argument for $r=1$ yields the estimate $O\left(k^{3 / 2}\right)$ as claimed.

Remark. The order in $k$ as $k \rightarrow \infty$ in the estimate for the constant $c$ in (2.14), is not optimal. The optimal order is, probably, $O(1)$. For a ball, for instance, we can prove that $\left|v_{N}\right|=O(k)$, rather than $O\left(k^{3 / 2}\right)$ and $|v|=O(1)$,

rather than $O\left(k^{1 / 2}\right)$. This yields $c=O(k)$ as $k \rightarrow \infty$. The estimate based on the Cauchy inequality, used in our derivation, does not take into account possible cancellations during integration in (2.10) due to oscillations of the integrand for large $k$. The optimal orders are: 1) $O(1)$ for $|v|, 2) O(k)$ for $\left|v_{N}\right|$ , and 3) $O(1)$ for the cross section as $k \rightarrow \infty$. These conclusions can be also obtained from the geometrical optics approximation (see formula (150.16) in H.Hönl,A.Maue, K.Westpfahl, Theorie der Beugung, Springer Verlag, Berlin, 1961).

6. Let us formulate the result proved in [49].

Theorem 1. Under the assumption made in section 2.1, estimate (2.11) holds with the constant $c>0$ independent of $t$, where $t \in[1, \infty], D_{j} \subset B_{a}$, $\partial D_{j} \subset C^{2, \nu},\left\|\phi_{j}\right\|_{C^{2, \nu}} \leq \Phi_{\nu}$.

In section 3 the proof of estimate (2.14) is given for the case $t_{1}=t_{2}=\infty$ which is of interest in applications. In section 4 applications are discussed.

\section{Proof of the Stability Estimate (2.14)}

Let us assume that

$$
q_{j}(x)= \begin{cases}+\infty, & \text { in } D_{j}, \\ 0, & \text { in } D_{j}^{\prime}:=R^{n} \backslash D_{j}, n \leq 2 .\end{cases}
$$

This is the case discussed in section 2.4 (see formula (2.14)). We assume 
$n=3$ for definiteness. The argument is the same for $n \geq 2$.

There are three ways to prove estimate (2.14) under the assumption (3.1). One way is to take $t_{1}=t_{2}=+\infty$ in (2.11), and note that the right-hand side equals $\operatorname{co}\left(D_{1}, D_{2}\right)$ if $t_{1}=t_{2}=+\infty$. The second way, is to take $t_{1}=t_{2}=$ $t<\infty$, and then let $t \rightarrow+\infty$, and use formula (2.8) and estimates (2.6), (2.7). These estimates allow one to derive formula (2.10) from which estimate (2.14) follows. Estimate (2.14) is a particular form of estimate (11) for the case when $\min \left(t_{1}, t_{2}\right)=+\infty$. The third way is based on estimate (2.10). Let us use this way. We assume that the distance $\rho\left(D_{j}, D_{m}\right), j \neq m$, is much greater than the distance $\rho\left(D_{j}, \tilde{D}_{j}\right)$, where $\tilde{D}_{j}$ is the perturbed domain $D_{j}$. The number $J$ of the connected components of the domain $D$ is fixed and finite. Therefore, the input of the variation of $\partial D$ in the scattering amplitude is of the order of magnitude of the input of the variation of $\partial D_{j}, 1 \leq j \leq J$. Therefore, one may use formula (10) assuming that $\partial D$ has one connected component $\partial D_{1}$, and $\partial D_{2}:=\partial \tilde{D}_{1}$ is a small variation of $\partial D_{1}$ in the sense that $\rho\left(D_{1}, D_{2}\right)$ is small. It follows from (2.10) that

$$
\begin{gathered}
\left|A\left(\alpha^{\prime}, \alpha, k\right)\right| \leq \frac{1}{4 \pi} \int_{\Gamma_{1}^{\prime}}\left|u_{1 N}(s, \alpha, k) u_{2}\left(s,-\alpha^{\prime}, k\right)\right| d s \\
+\int_{\Gamma_{2}^{\prime}}\left|u_{1}(s, \alpha, k) u_{2 N}\left(s,-\alpha^{\prime}, k\right)\right| d s:=I_{1}+I_{2},
\end{gathered}
$$

where $\Gamma_{1}^{\prime}$ is the part of $\Gamma_{1}$ which lies outside $D_{2}$, and $\Gamma_{2}^{\prime}$ is the part of $\Gamma_{2}$ which lies outside $D_{1}$.

One can use the following estimates

$$
\begin{gathered}
\gamma:=\max _{j=1,2} \sup _{s \in \Gamma_{j} ; \beta \in S^{2} ; 0<k_{1} \leq k \leq k_{2}<\infty}\left|u_{j N}(s, \beta, k)\right| \leq c, \\
\max _{j=1,2} \sup _{s \in \Gamma_{j}^{\prime} ; \beta \in S^{2} ; 0<k_{1} \leq k \leq k_{2}<\infty}\left|u_{j+1}(s, \beta, k)\right| \leq c \rho\left(D_{1}, D_{2}\right), \quad u_{3}:=u_{1},
\end{gathered}
$$


and formula (3.2), to get the desired estimate (2.14). Let us discuss estimates (3.3) and (3.4). The constant $c$ in (3.3) and (3.4) depends on the parameters $k_{1}, k_{2}, a$, and on the parameter $\Phi_{\nu}$, which is introduced in section 2.1, and which describes the smoothness of the boundary: $\left\|\phi_{j}\right\|_{C^{2, \nu}} \leq \Phi_{\nu}$. This constant does not depend on the particular choice of $D_{j}$. Let us prove the last claim. Suppose on the contrary, that there exists a sequence $D_{j n}$ of the obstacles $D_{j n} \subset B_{a},\left\|\phi_{j n}\right\|_{C^{2, \nu}} \leq \Phi_{\nu}$, such that $\gamma_{n} \geq c_{n}, c_{n} \rightarrow \infty$, where $c_{n}$ are the constants in (3.3), (3.4), and $\gamma_{n}$ is $\gamma$ for the obstacle $D_{j n}, n=1,2, \ldots$. By the Arzela-Ascoli compactness theorem one can assume that

$$
\phi_{j n} \stackrel{C^{2, \nu^{\prime}}}{\rightarrow} \psi_{j}, \quad 0<\nu^{\prime}<\nu, \quad u_{j n} \stackrel{H_{l o c}^{2}}{\rightarrow} u_{j}, \quad n \rightarrow \infty,
$$

where $u_{j}$ is the scattering solution corresponding to the limiting configuration of the surfaces $\Gamma_{1}, \Gamma_{2}$. For fixed surfaces $\Gamma_{1}$ and $\Gamma_{2}$, estimates (3.3) and (3.4) hold [53].

Note that it is sufficient to prove estimate (3.3). Indeed,

$$
\begin{aligned}
\left|u_{1}(s, \beta, k)\right|=\mid u_{1}(s, \beta, k) & -u_{1}(\tilde{s}, \beta, k)|\leq \sup | u_{1 N}(s, \beta, k)|| s-\tilde{s} \mid \\
& \leq c \rho\left(D_{1}, D_{2}\right),
\end{aligned}
$$

where $s \in \Gamma_{2}^{\prime}, \tilde{s} \in \Gamma_{1}, u_{1}(\tilde{s}, \beta, k)=0$, and the segment $\tilde{s} s$ is directed along the normal to $\Gamma_{2}^{\prime}$. A similar argument is valid for $u_{2}(s, \beta, k)=0, s \in \Gamma_{1}^{\prime}$.

If $\Gamma_{j n} \rightarrow \Gamma_{j}$ in the sense $\phi_{j n} \stackrel{C^{2, \nu^{\prime}}}{\rightarrow} \psi_{j}$ as $\left.n \rightarrow \infty\right)$, then $u_{j N n} \rightarrow u_{j N}$ as $n \rightarrow \infty$ (uniformly in $s \in \Gamma_{j}$ and in the parameters $\beta \in S^{2}, k \in\left[k_{1}, k_{2}\right]$, $\left.0<k_{1}<k_{2}<\infty\right)$, so that $\gamma_{n} \rightarrow \gamma$ as $n \rightarrow \infty$. Here $\gamma$ is the number defined by the left-hand side of (3.3) with $u_{j}$ corresponding to the limiting surfaces $\Gamma_{j}$. Since this $\gamma<\infty$, one obtains a contradiction: the inequality $\gamma_{n} \geq c_{n} \rightarrow+\infty$ contradicts to the equation $\gamma_{n} \rightarrow \gamma<\infty$. This contradiction 
proves that the constant $c$ in (3.3) and (3.4) does not depend on the particular choice of the obstacles $D_{j}$ as long as the two conditions are satisfied: $D_{j} \subset B_{a},\left\|\phi_{j}\right\|_{C^{2, \nu}} \leq \Phi_{\nu}$, and the parameters $a, \Phi_{\nu}, k_{1}$ and $k_{2}$ define the value of $c$ in (3.3), (3.4) and (2.14).

\section{Conclusions}

In connection with the problem of correlation effects in the quantum chaotic scattering and in mesoscopic systems, the consideration presented in sections 2 and 3 are of considerable interest. In particular, the estimate for the scattering amplitudes given by formula (2.14) is valid for the general case of singular potentials $q(x)$ supported in a compact region $D$. In this case the corresponding classical repeller $\Omega_{R}$ is chaotic, in general. So, result (2.14) means that the strong quantum correlations in the scattering amplitudes exist in some

region of parameters, even for classically chaotic (irregular) scattering, and are of the universal nature. The latter means that the quantum correlations in this region of parameters do not depend on the specific character of the resonance structure of the scattering amplitude. Estimate (2.14) includes the constant $c$ which actually depends on the system's parameters

$$
c=c\left(k_{1}, k_{2}, a, \Phi_{\nu}\right)
$$

That is why it is difficult to establish a direct relation between the region of parameters where estimate $(2.14)$ is valid, and the one $(\delta E>\Gamma>\Delta E)$ where the Ericson fluctuations are important.

The analytical and experimental investigations of the dependence (4.1) are of considerable interest for the further development of our understanding of the correlation effects in the processes of quantum chaotic scattering. The 
function (4.1) can be investigated, for example, in the experiments on the resonant tunneling in mesoscopic systems when the samples are prepared with small variation of the boundaries (scattering potential). Another possibility to investigate the correlation and fluctuation effects in quantum chaotic scattering can be realized in the microwave experiments (see, for example, [54]). The main idea, which is used in these experiments, is that the Schrödinger equation for a free particle reduces to the Helmholtz equation which describes the propagation of the classical waves. This correspondence was utilized in

[54] to investigate the role of fluctuations in the chaotic scattering. In our opinion, this method is rather promising: it allows one to imitate the ballistic regime in mesoscopic systems taking into account scattering, and to study the correlation effects in mesoscopic systems using a microwave technique.

\section{Acknowledgments}

AGR thanks LANL for support. GPB thanks Don Cohen, Gary Doolen and J.Mac Hyman of The Center for Nonlinear Studies, Los Alamos National Laboratory, for their hospitality. Also GPB gratefully acknowledges the partial support of the Linkage Grant 93-1602 from the NATO Special Programme Panel on Nanotechnology. 


\section{References}

1. Y. Imry, Europhys. Lett., 1 (1986) 249.

2. G. Timp, A.M. Chang, P. Mankiewich, R. Behringer, J.E. Cunningham, T.Y.Chang, R.E. Howard, Phys. Rev. Lett., 59 (1987) 732.

3. G. Timp, H.U. Baranger, P.de Vegvar, J.E. Cunningham, R.E. Howard, R. Behringer, P.M. Mankiewich, Phys. Rev. Lett., 60 (1988) 2081.

4. C.W.J. Beenakker, H. van Houten, Phys. Rev. Lett., 60 (1988) 2406.

5. C.J.B. Ford, S. Washburn, M. Büttiker, C.M. Knoedler, J.M. Hong, Phys. Rev. Lett., 62 (1989) 2724.

6. H.U. Baranger, A.D. Stone, Phys. Rev. Lett., 63 (1989) 414.

7. A.M. Chang, T.Y. Chang, H.U. Baranger, Phys. Rev. Lett., 63 (1989) 996.

8. H. Fukuyama, T. Ando (Eds.), Transport Phenomena in Mesoscopic Systems, Springer-Verlag, 1992.

9. Physics of Low-Dimensional Semiconductor Structures, Edited by P.Butcher, N.H. March, M.P. Tosi, Plenum Publishing Corporation, 1993.

10. Nanotechnology. Research and Perspectives, Edited by B.C. Crandall, 
J. Lewis, The MIT Press Cambridge, Massachusetts, London, 1992.

11. Physics of Nanostructures, Edited by J.H. Davies, A.R. Long, Proceedings of the Thirty-Eighth Scottsh Universities Summer School in Physics, St Andrews, 1991.

12. G. Bauer, F. Kuchar, H. Heinrich (Eds.), Low-Dimensional Electronic Systems. New Concepts, Springer-Verlag, 1992.

13. Semiconductors and Semimetals. Nanostructured Systems, Edited by M. Reed, Academic Press, Inc., 1992.

14. M.C. Gutzwiller, Physica D, 7 (1983) 341.

15. P. Gaspard, S.A. Rice, J. Chem. Phys., 90 (1989) 2225.

16. P. Gaspard, S.A. Rice, J. Chem. Phys., 90 (1989) 2242.

17. P. Gaspard, S.A. Rice, J. Chem. Phys., 90 (1989) 2255.

18. P. Gaspard, Proceedings of the International School of Physics "Enrico Fermi", Quantum Chaos, North-Holland, 1993, p. 307.

19. R. Blümel, U. Smilansky, Phys. Rev. Lett., 60 (1988) 477.

20. G. Troll, U. Smilansky, Physica D, 35 (1989) 34. 
21. R. Blümel, U. Smilansky, Physica D, 36 (1989) 111.

22. U. Smilansky, The Classical and Quantum Theory of Chaotic Scattering, in: Chaos and Quantum Physics, Les Houches, North-Holland, 1991, p. 370.

23. T. Ericson, Phys. Rev. Lett., 5 (1960) 430.

24. T. Ericson, Annals of Physics, 23 (1963) 390.

25. D.M. Brink, R.O. Stephen, Phys. Lett., 5 (1963) 77.

26. T. Ericson, T. Mayer-Kuckuk, Ann. Rev. Nucl. Sci., 16 (1966) 183.

27. W.H. Miller, J. Chem. Phys., 55 (1971) 3150.

28. W.H. Miller, Adv. Chem. Physics, 25 (1974) 66.

29. Mesoscopic Phenomena in Solids, Editors B.L. Altshuler, P.A. Lee, R.A. Webb, North-Holland, 1991.

30. E.E. Mendez, E. Calleja, C.E.T. Goncalves da Silva, L.L. Chang, W.I. Wang, Phys. Rev. B, 33 (1986) 7368.

31. J. Söderström, T.G. Andersson, J. Westin, Superlattices and Microstructures, 3 (1987) 283.

32. V.A. Chitta, C. Kutter, R.E.M. de Bekker, J.C. Maan, S.J. Hawksworth, 
J.M. Chamberlain, M. Henini, G. Hill, J. Phys.: Condens. Matter, 6 (1994) 3945 .

33. L.D. Landay, E.M. Lifshitz, Quanyum Mechanics, 2nd ed., Pergamon, New York, 1965.

34. C.E. Porter, Statistical Priperties of Spectra, Academic Press, New Your, 1965 .

35. B. Eckhardt, Phys. Reports, 163 (1988) 205.

36. B. Eckhardt, Physica D, 33 (1988) 89.

37. C. Gérard, J. Sjöstrand, Comm. Math. Phys., 108 (1987) 391.

38. B.V. Chirikov, Phys. Reports, 52 (1979) 1183.

39. M.V. Berry, ed., Dynamical Chaos, Cambridge University Press, 1988.

40. L.E. Reichl, The Transition to Chaos, Springer-Verlag, 1992.

41. Bibliography on Chaos, complited by Zhang Shu-yu, World Scientific, 1991.

42. V.M. Alekseev, Math. USSR, Sbornik, 5 (1968) 73; 6 (1968) 505; 7 (1969) 1. 
43. J. Moser, Stable and Random Motions in Dynamical Systems, Prinston Univ. Press, Princeton, NJ, 1973.

44. C. Grebogi, E. Ott, J.A. Yorke, Physica D, 7 (1983) 181.

45. W.H. Miller, J. Chem. Phys., 56 (1972) 38.

46. M.C. Gutzwiller, J. Math. Phys., 8 (1967) 1979; 10 (1969) 1004; 11 (1970) 1791; Phys. Rev. Lett., 45 (1980) 150; Physica D 5 (1982) 183; J. Phys. Chem., 92 (1988)3154.

47. A.G. Ramm, Izvestiya Vuzov, Mathematics, N5 (1965) 124; Math. Rev., $32 \# 7993$.

48. A.G. Ramm, J. Math. Anal. Appl., 84 (1981) 256.

49. A.G. Ramm, Stability Estimates for Obstacle Scattering, 1993.

50. A.G. Ramm, Multidimensional Inverse Scattering Problems, Longman/Wiley, New York, 1992.

51. A.G. Ramm, Appl. Math. Lett., 6,N5, (1993) 85.

52. A.G. Ramm, Acta Appl. Math., 28, N1, (1992) 1.

53. A.G. Ramm, Scattering by Obstacles, Reidel, Dordrecht, 1986. 
54. E. Doron, U. Smilansky, A. Frenkel, Proceedings of the International School of Physics "Enrico Fermi", Quantum Chaos, North-Holland, 1993, p. 399. 